\title{
The monocyte to high-density lipoprotein cholesterol ratio and outcomes in type 2 diabetes mellitus patients with non-ST- segment elevation acute coronary syndrome
}

\author{
Chen $\mathrm{Li}^{1 \#}$, Hualin Fan ${ }^{2 \#}$, Yuanhui Liu ${ }^{3}$, Lihuan Zeng ${ }^{4}$, Pengyuan Chen ${ }^{1}$, Chongyang Duan ${ }^{5}$, \\ Huasheng Liang', Pengcheng $\mathrm{He}^{1,2,3,4} \wedge$
}

${ }^{1}$ Department of Cardiology, Guangdong Provincial People's Hospital of Nanhai Hospital, the Second Hospital of Nanhai District Foshan City, Foshan, China; ${ }^{2}$ School of Medicine, South China University of Technology, Guangzhou, China; ${ }^{3}$ Department of Cardiology, Guangdong Cardiovascular Institute, Guangdong Provincial Key Laboratory of Coronary Heart Disease Prevention, Guangdong Provincial People's Hospital, Guangdong Academy of Medical Sciences, Guangzhou, China; ${ }^{4}$ The Second School of Clinical Medicine, Southern Medical University, Guangzhou, China; ${ }^{5}$ Department of Biostatistics, School of Public Health, Southern Medical University, Guangzhou, China

Contributions: (I) Conception and design: C Li, P He; (II) Administrative support: P He; (III) Provision of study materials or patients: H Fan, Y Liu; (IV) Collection and assembly of data: L Zeng, P Chen; (V) Data analysis and interpretation: C Duan, C Li, H Liang; (VI) Manuscript writing: All authors; (VII) Final approval of manuscript: All authors.

\#These authors contributed equally to this work.

Correspondence to: Pengcheng He, MD, PhD. Department of Cardiology, Guangdong Cardiovascular Institute, Guangdong Provincial Key Laboratory of Coronary Heart Disease Prevention, Guangdong Provincial People’s Hospital, Guangdong Academy of Medical Sciences, Guangzhou 510100, China. Email: gdhpc100@126.com.

Background: The monocyte to high-density lipoprotein cholesterol ratio (MHR) has been demonstrated as a new marker of inflammation. However, at present, the prognostic value of MHR in type 2 diabetes mellitus (T2DM) accompanied with non-ST-segment elevation acute coronary syndrome (NSTE-ACS) undergoing percutaneous coronary intervention (PCI) is unclear.

Methods: T2DM patients with NSTE-ACS undergoing PCI were consecutively enrolled from January 1, 2010 to December 31, 2014 and divided according to MHR value tertiles. Baseline, procedural, and followup data were collected. The primary outcomes were in-hospital major adverse clinical events (MACE). The prespecified secondary outcomes included any bleeding [as indicated by Bleeding Academic Research Consortium definition (BARC) grades 1-5] and death during follow-up.

Results: Of the 1,405 enrolled patients, the rates of in-hospital MACE $(0.2 \%, 0.2 \%$, and $1.3 \%, \mathrm{P}=0.043)$ and bleeding $(12.4 \%, 12.2 \%$, and $17.1 \%, \mathrm{P}=0.048)$ increased significantly in high MHR tertiles. After 1 year of follow-up, the rates of bleeding $(15.0 \%, 14.5 \%$, and $22.2 \%, \mathrm{P}=0.002)$ and all-cause death $(1.5 \%$, $1.7 \%$, and $4.3 \%, \mathrm{P}=0.010$ ) were higher in higher MHR tertiles. Our results also suggested that MHR was an independent predictor of in-hospital MACE [adjusted odds ratio $=8.36$; $95 \%$ confidence interval (CI): 1.57-44.47; $\mathrm{P}=0.013$ ] and long-term bleeding (adjusted hazard ratio $=1.21 ; 95 \% \mathrm{CI}$ : $1.07-1.37 ; \mathrm{P}=0.002$ ). Receiver-operating characteristic curve analysis indicated that MHR $>0.022$ had a sensitivity of $75.0 \%$ and specificity of $72.7 \%$ for predicting in-hospital MACE [area under the curve (AUC) $=0.722$; $95 \%$ CI: $0.51-$ 0.933; $\mathrm{P}=0.040]$. Furthermore, Kaplan-Meier curves showed that a higher risk of all-cause death in longterm follow-up was prevalent in patients with high MHR $(\mathrm{P}=0.033)$.

Conclusions: The increased level of MHR was related to in-hospital MACE and long-term bleeding events in T2DM patients with NSTE-ACS undergoing PCI.

$\wedge$ ORCID: 0000-0001-7706-7105. 
Keywords: Monocyte to high-density lipoprotein cholesterol ratio (MHR); non-ST-segment elevation acute coronary syndrome (NSTE-ACS); type 2 diabetes mellitus (T2DM); outcomes

Submitted Aug 19, 2021. Accepted for publication Oct 14, 2021.

doi: $10.21037 / \mathrm{atm}-21-4876$

View this article at: https://dx.doi.org/10.21037/atm-21-4876

\section{Introduction}

Despite the significant improvements of reperfusion strategies and antithrombotic therapy in recent years, acute coronary syndrome (ACS) remains the primary cause of mortality and morbidity worldwide (1). Diabetes is reported to be an independent risk factor for cardiovascular disease (CVD) and led to a high incidence of repeated hospitalization and poor prognoses $(2,3)$. A study of 1,612 patients with coronary heart disease followed up for 2.8 years undergoing PCI showed $6.4 \%$ patients died, and mortality risk increased with increasingly abnormal FG status (4). Diabetic suffering from ACS undergoing percutaneous coronary intervention (PCI) can have a high residual risk. Therefore, the early identification of high-risk patients is essential for better clinical strategies, in order to avoid cardiovascular events.

Previous studies have suggested that oxidative stress and inflammation are involved in the pathogenesis of all phases of atherosclerosis (5-7). It was reported that white blood cell (WBC) count and its subtypes are related to increased cardiovascular risk. For example, previous findings suggest that monocytes were particularly important in the pathogenesis of atherosclerosis owing to their ability to secrete pro-inflammatory and pro-oxidative cytokines $(8,9)$.

The monocyte to high-density lipoprotein cholesterol ratio (MHR) has been known as a prognostic predictor and new marker of CVD $(10,11)$. At present, the value of MHR in predicting the long-term prognosis of diabetic patients with non-ST-segment elevation acute coronary syndrome (NSTE-ACS) has not been investigated. Our study aims to evaluate the association between MHR and clinical outcomes in NSTE-ACS patients with type 2 diabetes mellitus (T2DM) undergoing PCI. We present the following article in accordance with the STROBE reporting checklist (available at https://dx.doi.org/10.21037/atm-21-4876).

\section{Methods}

\section{Experiment design and patients}

Our study was conducted based on a large cohort study that included 8,197 adults undergoing PCI for NSTE-ACS (12). Data on the clinical history, demographic features, inhospital management, physical examination, and outcomes of patients were fully described in our previous reports (12). In this study, of the 8,197 included patients, 243 patients were readmitted to the hospital. According to the exclusion criteria, 3,349 patients were excluded because of missing monocyte or high-density lipoprotein cholesterol (HDL-C) examination on admission. Furthermore, 90 patients with nosocomial infection, 45 patients with intra-aortic balloon pump, and 14 patients with PCI information incomplete were also excluded. A total of 3,051 patients were not diagnosed with T2DM. Finally, 1,405 patients were enrolled in this study (Figure 1).

All procedures performed in this study involving human participants were in accordance with the Declaration of Helsinki (as revised in 2013). The study was approved by the Institutional Ethics Committee of Guangdong Provincial People's Hospital of No. GDREC2016210H (R1).

\section{Data collection and processing}

Blood samples obtained immediately from all patients on admission before PCI were collected in this study. An automated blood cell counter (Sysmex XE-5000) purchased from Japan was used to analyze the following indicators of hemoglobin content: WBC, lymphocytes, neutrophils, monocytes, and platelets. A catalase assay was applied to analyze the serum HDL-C and other biochemical parameters using an automatic biochemical analyzing system (Beckman Coulter, USA). The MHR was obtained by dividing the monocyte count by the HDL-C count. Data were collected from the hospital by trained study coordinators.

\section{Clinical outcomes}

The primary outcomes were in-hospital major adverse clinical events (MACE). The prespecified secondary outcomes included any bleeding [as indicated by Bleeding 
All patients diagnosed as NSTE-ACS between January 1, 2010, to December 31, $2014(n=8,197)$
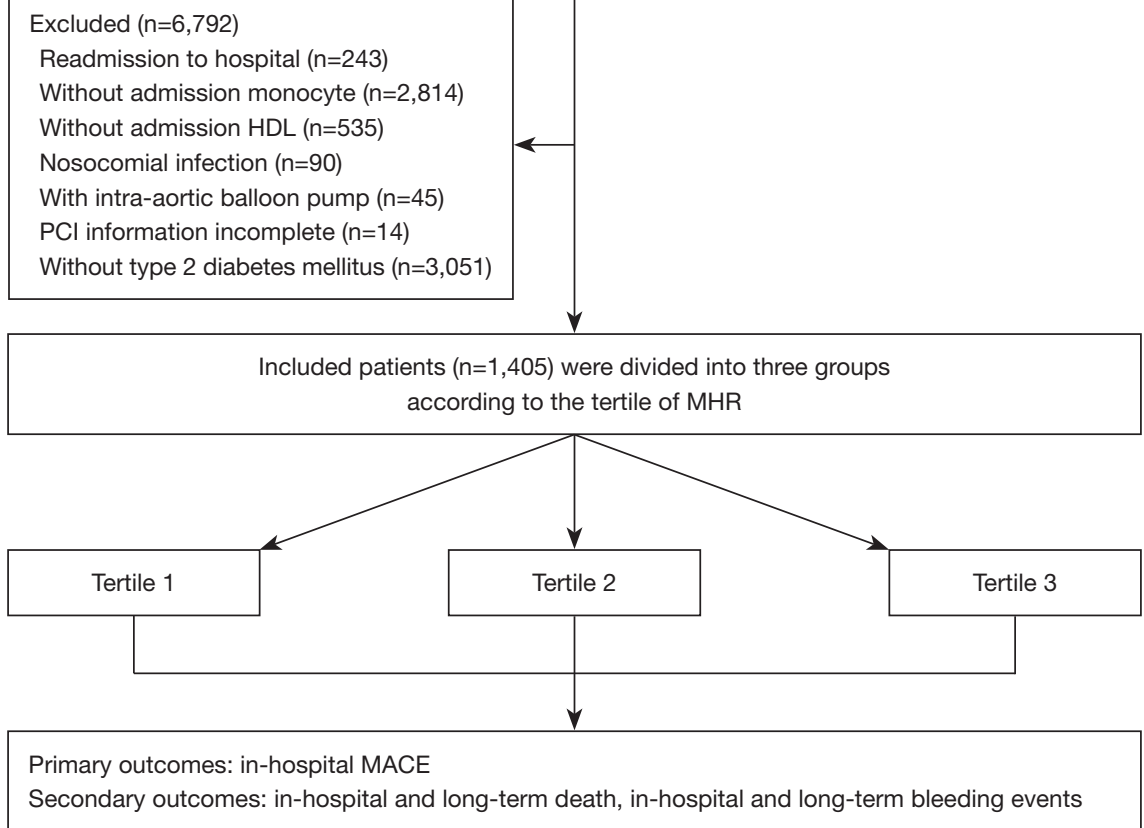

Figure 1 The flowchart presents the selection criteria used in the study and the clinical layout of the study population. NSTE-ACS, nonST-segment elevation acute coronary syndrome; MACE, major adverse cardiac event; PCI, percutaneous coronary intervention; MHR, monocyte to high-density lipoprotein cholesterol ratio.

Academic Research Consortium definition (BARC) grades 1-5] and death during follow-up (13). MACE was defined as a combination of non-fatal myocardial infarction (MI), stroke, all-cause death, and target vessel revascularization (TVR). Major bleeding was indicated by the BARC definition (grades 3-5) (13). Follow-up data was collected by trained nurses via telephone interview and outpatient visit.

\section{Statistical analysis}

The level of MHR was modeled as a continuous variable and in tertiles. Data were shown as mean and standard deviation, interquartile range and median, or proportions. Continuous data were compared using the $t$-test. Categorical data were expressed as a percentage, and were compared using Fisher's exact test and the $\chi^{2}$ test. Univariable and multivariable Cox proportional hazard regressions were conducted to obtain the odds ratio (OR) or hazard ratio (HR) and $95 \%$ confidence interval (CI), and to identify the relationship between the MHR (as a categorical or continuous variable) and clinical outcomes. Survival curves were constructed using the Kaplan-Meier method during follow-up for the MHR, and the log-rank test was used to perform statistical assessment. In the present study, SAS (version 9.4, SAS Institute, USA) was used to analyze the data based on existing cases without missing data. A two-sided $\mathrm{P}$ value $<0.05$ was considered to indicate statistical significance.

\section{Results}

\section{Baseline clinical characteristics}

The baseline characteristics results of the patient group based on the MHR tertile groups are presented in Table 1. In total, 1,405 patients were included, with an average age of 64.2 (10.4) years. Of these patients, 972 (69.2\%) were males. The differences between the groups in terms of gender, weight, smoking, and history of MI and atrial fibrillation were statistically significant. There were no significant differences in previous medications among the different triads of MHR. We found that increased WBC, neutrophil, lymphocyte, monocyte, and platelet (PLT), as 
Table 1 Baseline clinical characteristics of infective endocarditis patients according to the tertile of MHR

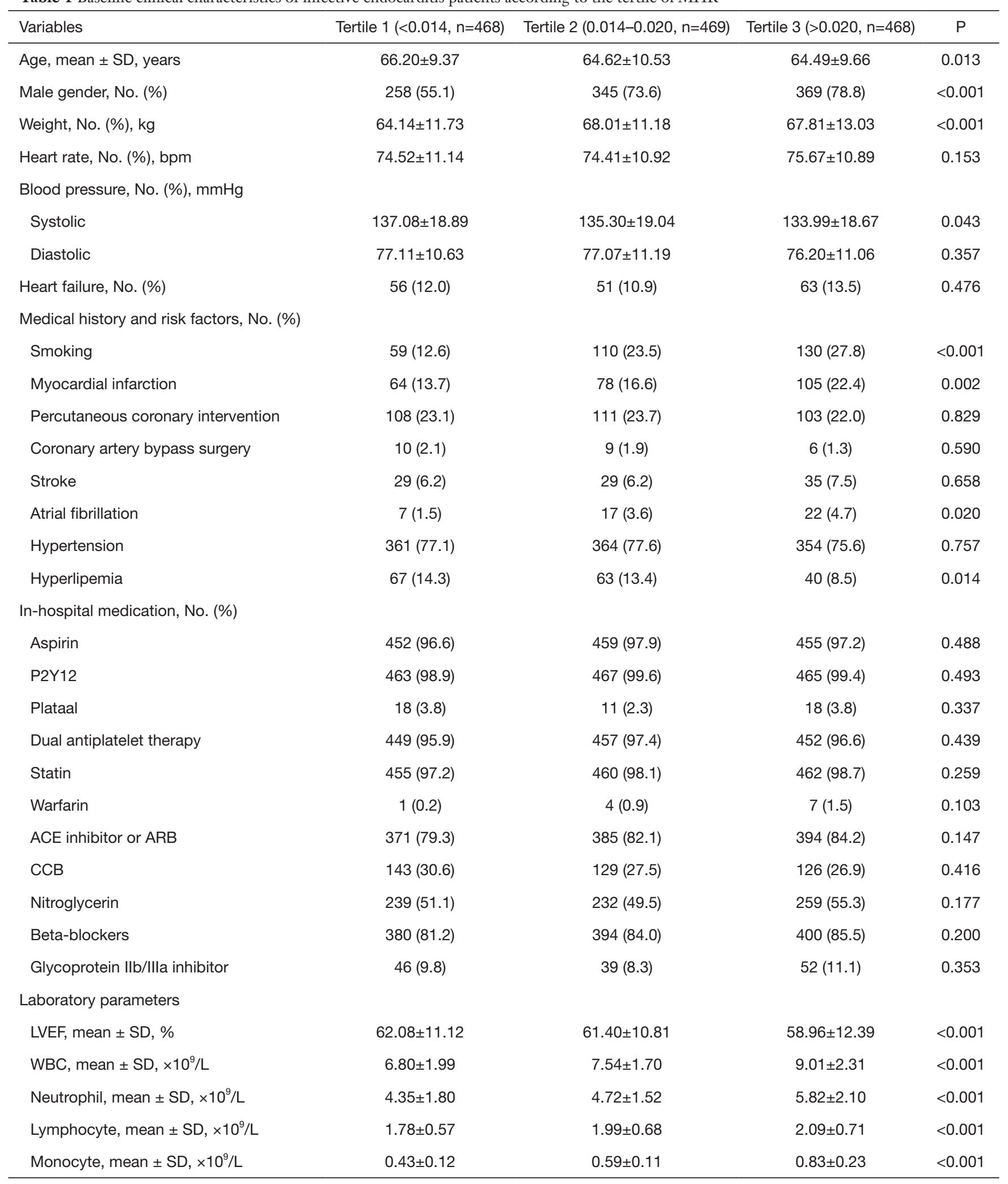

Table 1 (continued) 
Table 1 (continued)

\begin{tabular}{|c|c|c|c|c|}
\hline Variables & Tertile $1(<0.014, \mathrm{n}=468)$ & Tertile $2(0.014-0.020, n=469)$ & Tertile 3 (>0.020, $n=468)$ & $\mathrm{P}$ \\
\hline $\mathrm{PLT}$, mean $\pm \mathrm{SD}, \times 10^{9} / \mathrm{L}$ & $213.40 \pm 59.01$ & $217.92 \pm 57.71$ & $235.32 \pm 74.30$ & $<0.001$ \\
\hline $\mathrm{PDW}$, mean $\pm \mathrm{SD}, \mathrm{fL}$ & $15.49 \pm 2.17$ & $15.45 \pm 2.20$ & $15.44 \pm 2.21$ & 0.944 \\
\hline $\mathrm{TG}$, mean $\pm \mathrm{SD}, \mathrm{mmol} / \mathrm{L}$ & $2.01 \pm 2.02$ & $1.85 \pm 1.37$ & $1.89 \pm 1.35$ & 0.275 \\
\hline $\mathrm{HDL}-\mathrm{C}$, mean $\pm \mathrm{SD}, \mathrm{mmol} / \mathrm{L}$ & $1.11 \pm 0.32$ & $0.93 \pm 0.17$ & $0.79 \pm 0.16$ & $<0.001$ \\
\hline $\mathrm{LDL}-\mathrm{C}$, mean $\pm \mathrm{SD}, \mathrm{mmol} / \mathrm{L}$ & $2.74 \pm 0.98$ & $2.60 \pm 0.94$ & $2.47 \pm 0.86$ & $<0.001$ \\
\hline Serum creatinine, mean $\pm \mathrm{SD}, \mu \mathrm{mol} / \mathrm{L}$ & $97.12 \pm 87.65$ & $99.74 \pm 87.39$ & $108.21 \pm 77.52$ & 0.110 \\
\hline eGFR, mean $\pm \mathrm{SD}, \mathrm{mL} / \mathrm{min} / 1.73 \mathrm{~m}^{2}$ & $80.45 \pm 28.42$ & $81.53 \pm 29.51$ & $75.18 \pm 29.81$ & 0.002 \\
\hline
\end{tabular}

$\mathrm{ACE}$, angiotensin-converting enzyme; ARB, angiotensin receptor blockers; CCB, calcium channel blocker; LVEF, left ventricular ejection fraction; WBC, white blood cell; PLT, platelet; PDW, platelet distribution width; TG, total triglyceride; TC, total cholesterol; LDL-C, low-density lipoprotein cholesterol; HDL-C, high-density lipoprotein cholesterol; eGFR, estimated glomerular filtration rate; GRACE, Global Registry of Acute Coronary Events; SD, standard deviation

well as decreased total cholesterol (TC), HDL-C, lowdensity lipoprotein (LDL), detected glomerular filtration rate, and left ventricular ejection fraction were more prevalent in the highest tertile group.

\section{Clinical outcomes}

In this study, the incidence of MACE $(0.2 \%, 0.2 \%$, and $1.3 \%, \mathrm{P}=0.043)$ and any bleeding $(12.4 \%, 12.2 \%$, and $17.1 \%, \mathrm{P}=0.048$ ) was increased in patients with the highest MHR tertiles. However, among the three groups, no significant difference was noted in the in-hospital all-cause deaths $(0.0 \%, 0.0 \%$ and $0.4 \%, \mathrm{P}=0.135)$ and major bleeding $(1.1 \%, 1.3 \%$ and $1.5 \%, \mathrm{P}=0.845)$ (Table 2). In the univariate analysis, increased MHR was associated with in-hospital MACE. Furthermore, multivariate analysis indicated that the MHR (adjusted OR: 8.36; 95\% CI: 1.57-44.47; P=0.013) was an independent predictor of in-hospital MACE, after adjusting for other variables (Table 3). The multivariate Cox proportional hazard regression model showed that the MHR (adjusted HR: 1.21; 95\% CI: $1.07-1.37 ; \mathrm{P}=0.002$ ) was an independent predictor of long-term bleeding, after adjusting for other factors (Table 4).

Receiver-operating characteristic curve analysis indicated that MHR $>0.022$ had a sensitivity of $75.0 \%$ and specificity of $72.7 \%$ for predicting in-hospital MACE [area under the curve $(\mathrm{AUC})=0.722 ; 95 \% \mathrm{CI}: 0.51-0.933 ; \mathrm{P}=0.040]$
(Figure 2). Kaplan-Meier curves based on the cut-off values of MHR are shown in Figure 3. Patients with a high MHR were found to have a significantly higher long-term mortality rate $(33.48 \%$ vs. $13.71 \%, \mathrm{P}=0.003$, Figure 3$)$.

\section{Discussion}

In the present study, our findings demonstrated the predictive value of MHR in T2DM patients undergoing PCI for NSTE-ACS. It was noted that the MHR was independently related to in-hospital MACE or long-term bleeding events. Furthermore, our study revealed that MHR $>0.022$ had good discrimination in predicting inhospital MACE.

T2DM is a pro-inflammatory disease, and an enhanced inflammatory reaction at the site of implantation of stents after PCI. Compared with normal patients, DM patients have a higher incidence of composite clinical outcomes (14). Circulating monocytes, which function as a source of molecules and cytokines, lead to the activation of prothrombotic pathways and inflammation via interaction with platelets or endothelial cells. However, HDL-C can inhibit the migration and oxidation of macrophages and LDL molecules, respectively. Additionally, HDL-C promotes the outflow of cholesterol from these cells. HDL-C has been shown to inhibit the pro-inflammation and pro-oxidation of monocytes (15). 
Table 2 In-hospital and long-term clinical outcomes

\begin{tabular}{|c|c|c|c|c|}
\hline Outcome & Tertile $1(n=468)$ & Tertile $2(n=469)$ & Tertile $3(n=468)$ & $\mathrm{P}$ \\
\hline MACE & $1(0.2)$ & $1(0.2)$ & $6(1.3)$ & 0.043 \\
\hline Any bleeding & $58(12.4)$ & $57(12.2)$ & $80(17.1)$ & 0.048 \\
\hline Major bleeding & $5(1.1)$ & $6(1.3)$ & $7(1.5)$ & 0.845 \\
\hline \multicolumn{5}{|c|}{ Long-term outcome, No. (\%) } \\
\hline \multicolumn{5}{|l|}{30 days } \\
\hline Any bleeding & $58(12.4)$ & $58(12.4)$ & $81(17.3)$ & 0.043 \\
\hline Major bleeding & $4(0.9)$ & $6(1.3)$ & $7(1.5)$ & 0.659 \\
\hline Any bleeding & $62(13.2)$ & $63(13.4)$ & $87(18.6)$ & 0.035 \\
\hline Major bleeding & $6(1.3)$ & $7(1.5)$ & $10(2.1)$ & 0.562 \\
\hline Death & $3(0.6)$ & $2(0.4)$ & $10(2.1)$ & 0.021 \\
\hline \multicolumn{5}{|l|}{1 year } \\
\hline Any bleeding & $70(15.0)$ & $68(14.5)$ & $104(22.2)$ & 0.002 \\
\hline Major bleeding & $7(1.5)$ & $8(1.7)$ & $11(2.4)$ & 0.600 \\
\hline Death & $7(1.5)$ & $8(1.7)$ & $20(4.3)$ & 0.010 \\
\hline
\end{tabular}

MACE, major adverse cardiovascular event.

Table 3 Significant predictors of in-hospital MACEs in univariate and multivariate analyses

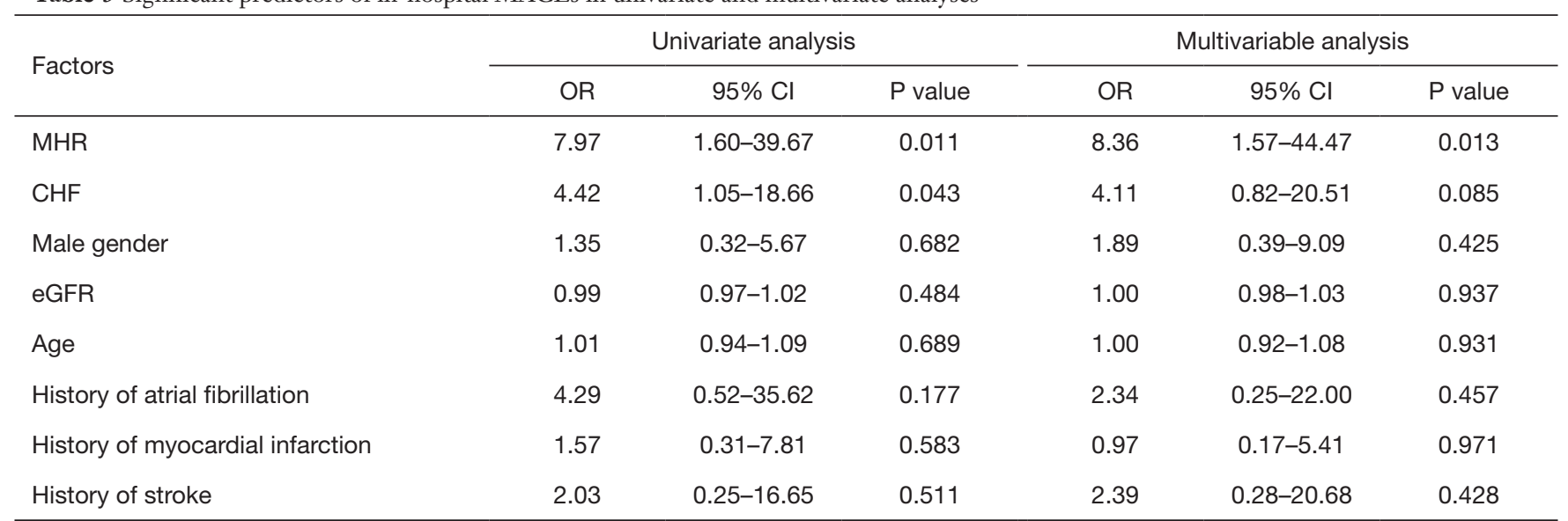

MACE, major adverse cardiac event; MHR, monocyte to high-density lipoprotein cholesterol ratio; CHF, chronic heart failure; eGFR, estimated glomerular filtration rate; OR, odds ratio; $\mathrm{Cl}$, confidence interval. 
Table 4 Significant predictors of any bleeding in univariable and multivariable Cox regression analyses

\begin{tabular}{|c|c|c|c|c|c|c|}
\hline Factors & \multicolumn{3}{|c|}{ Univariate analysis } & \multicolumn{3}{|c|}{ Multivariable analysis } \\
\hline MHR & 1.21 & $1.08-1.36$ & 0.001 & 1.21 & $1.07-1.37$ & 0.002 \\
\hline $\mathrm{CHF}$ & 1.15 & $0.83-1.60$ & 0.389 & 0.87 & $0.62-1.23$ & 0.432 \\
\hline Male gender & 1.24 & $0.98-1.57$ & 0.070 & 1.21 & $0.94-1.54$ & 0.135 \\
\hline Age & 1.03 & $1.01-1.04$ & $<0.001$ & 1.02 & $1.00-1.03$ & 0.009 \\
\hline History of atrial fibrillation & 1.87 & $1.15-3.05$ & 0.012 & 1.46 & $0.89-2.41$ & 0.135 \\
\hline History of myocardial infarction & 1.12 & $0.84-1.50$ & 0.422 & 1.10 & $0.82-1.47$ & 0.544 \\
\hline History of stroke & 1.24 & $0.82-1.89$ & 0.303 & 1.16 & $0.76-1.76$ & 0.493 \\
\hline
\end{tabular}

MHR, monocyte to high-density lipoprotein cholesterol ratio; CHF, chronic heart failure; eGFR, estimated glomerular filtration rate; HR, hazard ratio; $\mathrm{Cl}$, confidence interval.

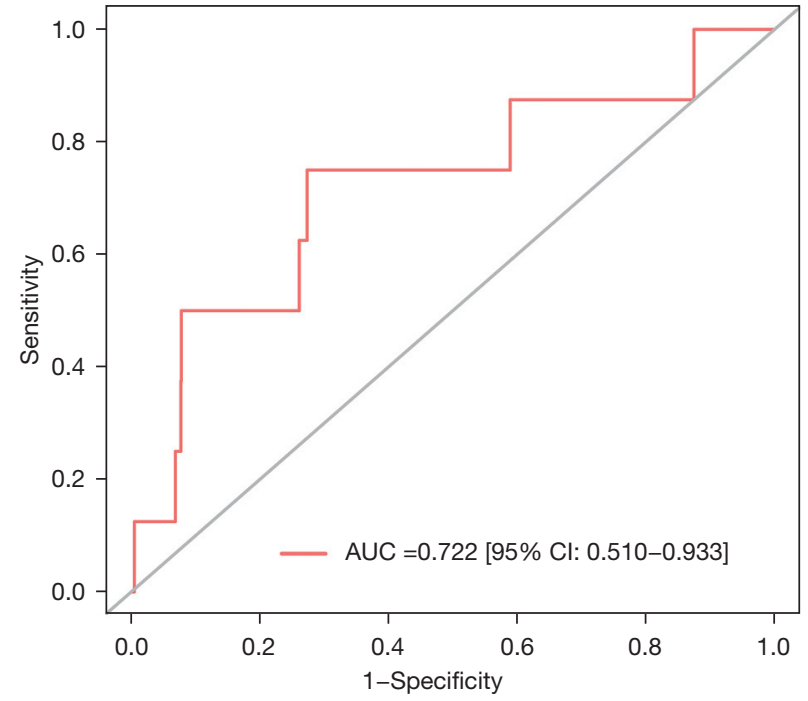

Figure 2 ROC curves analysis showing the predictive cut-off value of MHR for in-hospital MACE. ROC, receiver-operating characteristic; MHR, monocyte to high-density lipoprotein cholesterol ratio; MACE, major adverse cardiac events.

Increased MHR is prevalent in a variety of cardiovascular conditions, which is thought to contribute to the pathogenesis and progression of these diseases. Moreover, increased MHR was also demonstrated as a powerful predictive marker for the early diagnosis of cardiovascular diseases. A previous study conducted in patients revealed that the MHR can be regarded as an independent predictor of major cardiovascular events (16). MHR was reported to be correlated with long-term mortality and in-hospital and

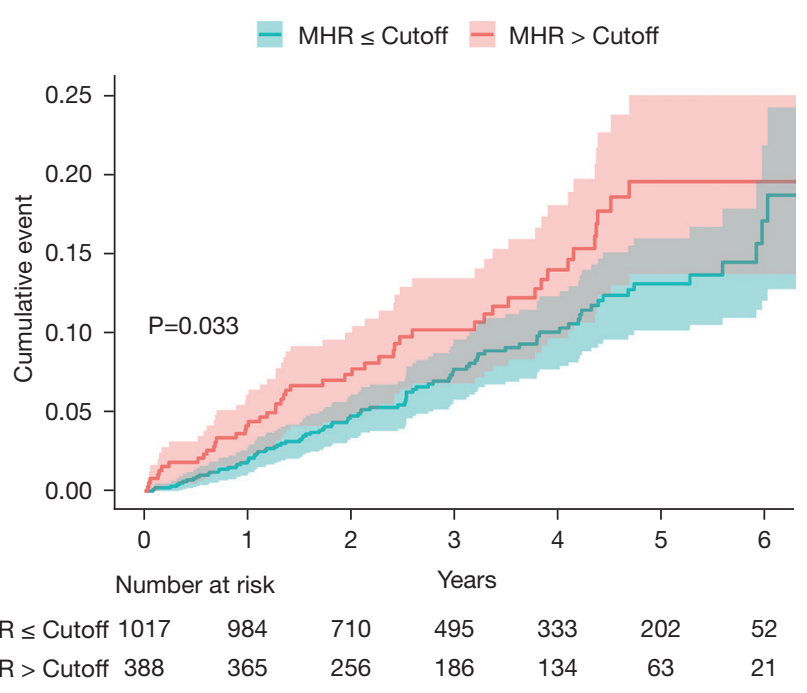

Figure 3 Kaplan-Meier estimated rates of all-cause death. Cumulative rate of long-term mortality in NSTE-ACS patients with and without a MHR >0.022. NSTE-ACS, non-ST-segment elevation acute coronary syndrome; MHR, monocyte to high-density lipoprotein cholesterol ratio.

MACE in ST-Segment Elevation Myocardial Infarction (STEMI) (17). Cetin et al. claimed that the MHR, as a new inflammatory marker, was related to cardiovascular events in patients with ACS (18). Another previous study demonstrated that MHR plays a role in the increased recurrence of atrial fibrillation in patients undergoing cryoballoon-based catheter ablation (19). Chen et al. reveal that MHR is both associated with basal thickness and 
progression of CIMT inpatients with T2DM, and logtransformed MHR was associated with elevated CIMT in diabetic but not non-diabetic patients (5). Although MHR is associated with multiple complications of diabetes, there was no difference with regard to MHR between patients with or without diabetic peripheral neuropathy in a study (20). MHR has also been used in the early diagnosis of acute ischemic stroke (21), contrast-induced nephropathy (22), hyperuricemia (23), and metabolic syndrome (24). Our study revealed the potential predictive value of MHR in T2DM patients undergoing PCI for NSTE-ACS. Our results illustrated that MHR was independently related to MACE and long-term bleeding events during hospitalization. Furthermore, MHR $>0.022$ was a notable predictor of inhospital MACE and long-term death. Thus, we assumed that MHR is a predictor of abnormal lipid metabolism and inflammation status in patients with NSTE-ACS and T2DM. Additionally, our findings in the present study are consistent with the previous findings. The etiology of NSTE-ACS is complicated, and involves an inflammatory reaction, alternated lipid metabolism, oxidation injury, and other pathological processes (25-27). Some potential prognostic marker as neutrophil to high-density lipoprotein ratio (NHR), monocyte lymphocyte ratio (MLR), neutrophil to lymphocyte ratio (NLR), Total antioxidant status (TAS), total oxidant status (TOS), oxidative stress index (OSI), and ischemia-modified albumin (IMA) have also been confirmed affect the prognosis of coronary artery disease (28-31).

In addition, it was noted that a high level of MHR was an independent risk factor of bleeding events. The precise role of high MHR in the increased bleeding risk of T2DM patients with NSTE-ACS is still unclear. A previous study has shown that higher monocyte counts in patients with atrial fibrillation are related to an increased risk of major bleeding. The underlying mechanism of this is excessive elevation in monocytes or abnormal function of monocytes may alter the strictly controlled local hemostasis towards a bleeding state. The ability of monocytes to produce matrix metalloproteinases may further amplify this process, thus overcoming the characteristics of procoagulant monocytes (32). Our findings support the idea that excessive monocytes may be associated with any bleeding events; however, no significant increase in major bleeding was observed in our study.

However, our study still has some limitations that should be noted. Firstly, there was no dynamic monitoring of MHR, so it was not clear whether the changes in MHR were related to the prognosis. Secondly, other oxidative stress and inflammation markers, such as uric acid and C-reactive protein, were not compared with the MHR. To further improve the validity and predictive power of our model, the sample size should be increased as much as possible, and interference of numerous confounding factors should be eliminated through multi-factor correction. Therefore, the relationship between dynamic MHR and prognosis, and the comparison between the MHR and classical inflammatory markers should be explored in future studies.

\section{Conclusions}

In summary, this study demonstrated that the MHR is an independent predictor of in-hospital MACE and long-term mortality in T2DM patients with NSTE-ACS undergoing PCI. Furthermore, it was revealed that increased levels of MHR were significantly related to bleeding events.

\section{Acknowledgments}

Funding: This work was supported by the Outstanding Young Talent Program of Guangdong Provincial People's Hospital (Grant No. KJ012019084 and KJ012019095), and the High-level Hospital Construction Project (Grant No. DFJH2020021).

\section{Footnote}

Reporting Checklist: The authors have completed the STROBE reporting checklist. Available at https://dx.doi. org/10.21037/atm-21-4876

Data Sharing Statement: Available at https://dx.doi. org/10.21037/atm-21-4876

Conflicts of Interest: All authors have completed the ICMJE uniform disclosure form (available at https://dx.doi. org/10.21037/atm-21-4876). The authors have no conflicts of interest to declare.

Ethical Statement: The authors are accountable for all aspects of the work in ensuring that questions related to the accuracy or integrity of any part of the work are appropriately investigated and resolved. All procedures performed in this study involving human participants were in accordance with the Declaration of Helsinki (as revised in 2013). The study was approved by the Institutional Ethics 
Committee of Guangdong Provincial People's Hospital of No. GDREC2016210H(R1) and informed consent was taken from all the patients.

Open Access Statement: This is an Open Access article distributed in accordance with the Creative Commons Attribution-NonCommercial-NoDerivs 4.0 International License (CC BY-NC-ND 4.0), which permits the noncommercial replication and distribution of the article with the strict proviso that no changes or edits are made and the original work is properly cited (including links to both the formal publication through the relevant DOI and the license). See: https://creativecommons.org/licenses/by-nc-nd/4.0/.

\section{References}

1. Lopez AD, Mathers CD, Ezzati M, et al. Global and regional burden of disease and risk factors, 2001: systematic analysis of population health data. Lancet 2006;367:1747-57.

2. Low Wang CC, Hess CN, Hiatt WR, et al. Clinical Update: Cardiovascular Disease in Diabetes Mellitus: Atherosclerotic Cardiovascular Disease and Heart Failure in Type 2 Diabetes Mellitus - Mechanisms, Management, and Clinical Considerations. Circulation 2016;133:2459-502.

3. El Sanadi CE, Ji X, Kattan MW. 3-point major cardiovascular event outcome for patients with T2D treated with dipeptidyl peptidase-4 inhibitor or glucagonlike peptide-1 receptor agonist in addition to metformin monotherapy. Ann Transl Med 2020;8:1345.

4. Muhlestein JB, Anderson JL, Horne BD, et al. Effect of fasting glucose levels on mortality rate in patients with and without diabetes mellitus and coronary artery disease undergoing percutaneous coronary intervention. Am Heart J 2003;146:351-8.

5. Chen JW, Li C, Liu ZH, et al. The Role of Monocyte to High-Density Lipoprotein Cholesterol Ratio in Prediction of Carotid Intima-Media Thickness in Patients With Type 2 Diabetes. Front Endocrinol (Lausanne) 2019;10:191.

6. Libby P. Inflammation in atherosclerosis. Nature 2002;420:868-74.

7. Fiechter M, Ghadri JR, Jaguszewski M, et al. Impact of inflammation on adverse cardiovascular events in patients with acute coronary syndromes. J Cardiovasc Med (Hagerstown) 2013;14:807-14.

8. Horne BD, Anderson JL, John JM, et al. Which white blood cell subtypes predict increased cardiovascular risk? J Am Coll Cardiol 2005;45:1638-43.
9. Arbel Y, Finkelstein A, Halkin A, et al. Neutrophil/ lymphocyte ratio is related to the severity of coronary artery disease and clinical outcome in patients undergoing angiography. Atherosclerosis 2012;225:456-60.

10. Moro-García MA, Echeverría A, Galán-Artímez MC, et al. Immunosenescence and inflammation characterize chronic heart failure patients with more advanced disease. Int $\mathbf{J}$ Cardiol 2014;174:590-9.

11. Colin S, Fanchon M, Belloy L, et al. HDL does not influence the polarization of human monocytes toward an alternative phenotype. Int J Cardiol 2014;172:179-84.

12. Chen JY, He PC, Liu YH, et al. Association of Parenteral Anticoagulation Therapy With Outcomes in Chinese Patients Undergoing Percutaneous Coronary Intervention for Non-ST-Segment Elevation Acute Coronary Syndrome. JAMA Intern Med 2019;179:186-94.

13. Mehran R, Rao SV, Bhatt DL, et al. Standardized bleeding definitions for cardiovascular clinical trials: a consensus report from the Bleeding Academic Research Consortium. Circulation 2011;123:2736-47.

14. Zhao X, Lan J, Yu X, et al. Primary Percutaneous Coronary Intervention in Patients With Type 2 Diabetes With Late/Very Late Stent Thrombosis and de novo Lesions: A Single-Center Observational Cohort Study of Clinical Outcomes and Influencing Factors. Front Cardiovasc Med 2021;8:653467.

15. Ucar FM. A potential marker of bare metal stent restenosis: monocyte count - to- HDL cholesterol ratio. BMC Cardiovasc Disord 2016;16:186.

16. Kanbay M, Solak Y, Unal HU, et al. Monocyte count/ HDL cholesterol ratio and cardiovascular events in patients with chronic kidney disease. Int Urol Nephrol 2014;46:1619-25.

17. Açıkgöz SK, Açıkgöz E, Şensoy B, et al. Monocyte to highdensity lipoprotein cholesterol ratio is predictive of inhospital and five-year mortality in ST-segment elevation myocardial infarction. Cardiol J 2016;23:505-12.

18. Cetin MS, Ozcan Cetin EH, Kalender E, et al. Monocyte to HDL Cholesterol Ratio Predicts Coronary Artery Disease Severity and Future Major Cardiovascular Adverse Events in Acute Coronary Syndrome. Heart Lung Circ 2016;25:1077-86.

19. Canpolat U, Çetin EH, Cetin S, et al. Association of Monocyte-to-HDL Cholesterol Ratio with Slow Coronary Flow is Linked to Systemic Inflammation. Clin Appl Thromb Hemost 2016;22:476-82.

20. Gökçay Canpolat A, Emral R, Keskin Ç, et al. Association of monocyte-to-high density lipoprotein-cholesterol 


\section{Page 10 of 10}

ratio with peripheral neuropathy in patients with Type II diabetes mellitus. Biomark Med 2019;13:907-15.

21. Bolayir A, Gokce SF, Cigdem B, et al. Monocyte/highdensity lipoprotein ratio predicts the mortality in ischemic stroke patients. Neurol Neurochir Pol 2018;52:150-5.

22. Sağ S, Yıldız A, Aydin Kaderli A, et al. Association of monocyte to HDL cholesterol level with contrast induced nephropathy in STEMI patients treated with primary PCI. Clin Chem Lab Med 2017;55:132-8.

23. Chen MQ, Shi WR, Shi CN, et al. Impact of monocyte to high-density lipoprotein ratio on prevalent hyperuricemia: findings from a rural Chinese population. Lipids Health Dis 2020;19:48.

24. Jialal I, Jialal G, Adams-Huet B, et al. Neutrophil and monocyte ratios to high-density lipoprotein-cholesterol and adiponectin as biomarkers of nascent metabolic syndrome. Horm Mol Biol Clin Investig 2020;41. doi: 10.1515/hmbci-2019-0070.

25. Hickman PE, McGill DA, Talaulikar G, et al. Prognostic efficacy of cardiac biomarkers for mortality in dialysis patients. Intern Med J 2009;39:812-8.

26. Kehl DW, Iqbal N, Fard A, et al. Biomarkers in acute myocardial injury. Transl Res 2012;159:252-64.

27. Xu L, Su Y, Zhao Y, et al. Melatonin differentially regulates pathological and physiological cardiac hypertrophy: Crucial role of circadian nuclear receptor

Cite this article as: Li C, Fan H, Liu Y, Zeng L, Chen P, Duan C, Liang H, He P. The monocyte to high-density lipoprotein cholesterol ratio and outcomes in type 2 diabetes mellitus patients with non-ST-segment elevation acute coronary syndrome. Ann Transl Med 2021;9(21):1627. doi: 10.21037/ atm-21-4876

\section{Li et al. MHR and outcomes in T2DM patients with NSTE-ACS}

ROR $\alpha$ signaling. J Pineal Res 2019;67:e12579.

28. Huang JB, Chen YS, Ji HY, et al. Neutrophil to highdensity lipoprotein ratio has a superior prognostic value in elderly patients with acute myocardial infarction: a comparison study. Lipids Health Dis 2020;19:59.

29. Fan Z, Li Y, Ji H, et al. Prognostic utility of the combination of monocyte-to-lymphocyte ratio and neutrophil-to-lymphocyte ratio in patients with NSTEMI after primary percutaneous coronary intervention: a retrospective cohort study. BMJ Open 2018;8:e023459.

30. Çiçek G, Açıkgoz SK, Bozbay M, et al. Neutrophillymphocyte ratio and platelet-lymphocyte ratio combination can predict prognosis in patients with STsegment elevation myocardial infarction undergoing primary percutaneous coronary intervention. Angiology 2015;66:441-7.

31. Demirbag R, Rabus B, Sezen Y, et al. The plasma and tissue oxidative status in patients with coronary artery disease: oxidative stress and coronary artery disease. Turkish J Thoracic Cardiovasc Surg. 2010;18:79-82.

32. Shahid F, Rahmat NA, Lip GYH, et al. Prognostic implication of monocytes in atrial fibrillation: The West Birmingham Atrial Fibrillation Project. PLoS One 2018;13:e0200373.

(English Language Editor: A. Kassem) 